\title{
Influence of Bio Fertilizer on Growth and Yield Parameters of Tomato Plants ${ }^{\dagger}$
}

\author{
P. Sajeesh ${ }^{1}$, Yucinda K.Y.Y. ${ }^{1}$, P. Paulraj ${ }^{1}$, K.P. Sajna ${ }^{1}$, T. Ketharin ${ }^{1}$, P.T. Mohamed Javad ${ }^{1}$ \\ 1 School of Bioscience, Faculty of Medicine, Bioscience and Nursing, MAHSA University, Jenjarom, Selangor, Malaysia \\ * Correspondence: Not available; \\ $\dagger$ Presented at International e-Conference on Bioengineering for Health and Environment (ICBHE 2020)
}

Received: 5.07.2020; Revised: 10.07.2020; Accepted: 12.07.2020; Published: 15.07.2020

\begin{abstract}
Tomato is a major food that has many benefits to human health. The risk of getting disorders such as heart disease, cancer, and diabetes can be reduced by consuming tomato. The aim of this study is to measure the nutritional properties of tomato plants using various cultivation methods. On the other hand, this is also comparing which type of fertilizer, either chemical fertilizer or natural fertilizer, is producing better results in planting tomato. Phosphorus solubilizing bacteria (PSB) is isolated from the rhizosphere soil using the Pikovskaya medium and Modified Aleksandrow medium. Instead of chemical fertilizer, the efficiency of phosphate solubilize will be evaluated in the presence of eggshells and bones. This study starts with the collection of rhizosphere and non-rhizosphere soil for tomato planting. The land of cultivation for planting well estimated for the growth of tomato. For further investigation, the leaves and fruits obtained from the tomato plants will be dried and ground into powder form. The leaf is used for the estimation of chlorophyll. In contrast, the fruit powder is used for the analysis of antioxidant activity. The result showed that the growth and yield parameters of the plant increases with natural fertilizer compare to chemical fertilizer. This can be concluded that natural fertilizers are more preferable in the agriculture industry as they are environmentally friendly, which does not produce side effects in either the soils or the crops.
\end{abstract}

Keywords: chemical fertilizer; rhizosphere; chlorophyll; yield; environmentally friendly.

(C) 2020 by the authors. This article is an open-access article distributed under the terms and conditions of the Creative Commons Attribution (CC BY) license (https://creativecommons.org/licenses/by/4.0/).

\section{Funding}

This research received no external funding.

\section{Acknowledgments}

This research has no acknowledgment.

\section{Conflicts of Interest}

The authors declare no conflict of interest. 